\title{
Trombosis venosa cerebral profunda puerperal
}

\author{
E. Franco, E. Gil-Néciga, E. Salinas, R. Morales a , E. Martínez, A. Donaire
}

\author{
DEEP CEREBRAL VENOUS THROMBOSIS AT PUERPERIUM
}

\begin{abstract}
Summary. Clinical case. Two weeks after an induced delivery with oxytocin, a 29 year-old healthy primipara started with headache, fever and confusion. The brain CT showed diffuse bilateral thalamic hypodensities and an 'empty delta sign' at the level of Galen's vein. The angiographic studies and the brain MR were diagnostic for deep cerebral venous thrombosis (DCVT). In blood tests a lupus anticoagulant was detected and the patient was treated with warfarin. In the next days she fully recovered. Conclusions. Isolated DCVT is uncommon. The existence of a bilateral and diffuse thalamic ischemia should suggest a Galen's vein thrombosis, mainly if it appears in the course of well known hypercoagulative states such as pregnancy and puerperium. The good outcome of our patient has already been reported in some other cases of DCVT and can be explained by the existence of an efficient complementary venous circulation. This mild form of DCVT is unusual as it often evolves with a great mortality and discapacity [REV NEUROL 1999; 29: 722-4].
\end{abstract}

Key words. Deep cerebral venous thrombosis. Lupus anticoagulant. Puerperium. Thalamic infarction.

\section{INTRODUCCIÓN}

La trombosis venosa cerebral profunda (TVCP) sigue siendo una entidad rara. Tan sólo el $1 \%$ de las trombosis venosas cerebrales (TVC) se presentan con oclusión aislada de las venas cerebrales profundas [1] y la mayoría de los casos descritos han ocurrido en niños, particularmente en recién nacidos. Se han publicado medio centenar de casos adultos desde la primera descripción [2]. El puerperio, el embarazo, la toma de anticonceptivos y los trastornos de coagulabilidad son, como en el resto de las trombosis venosas, los factores predisponentes más comunes [3].

Las descripciones clásicas de esta entidad coinciden en la presentación: cuadroneurológico agudograve, con disminución del nivel de conciencia, rigidez de descerebración y signos extrapiramidales. Es habitual que se produzca la muerte en pocos días, o secuelas graves como mutismo acinético, demencia, movimientos atetoides bilaterales, parálisis de la mirada vertical y distonía, [1,4]. Sin embargo, recientemente se han descrito formas benignas de TVCP, cuya manifestación fundamental consiste en un cuadro confusional reversible [5-7].

Signos radiológicos de edema o infarto bitalámico extenso deben hacer pensar en una TVCP. Un signo ‘delta vacío’ en la TAC con contraste a nivel de la vena de Galeno apoya con consistencia el diagnóstico [3,4,8]. Los cortes sagitales en la RM cerebral pueden permitir visualizar la trombosis a nivel de las venas cerebrales profundas $[3,4,9]$ y la angiografía en fases venosas con ausencia de relleno del sistema venoso profundoes diagnóstica [3,7-9].

\section{CASO CLÍNICO}

Mujer primípara de 29 años, con antecedentes de alergia al polen y a algunos conservantes alimenticios. Durante su embarazo se detectaron ocasionalmente cifras tensionales elevadas, habiendo realizado temporal-

Recibido: 16.10.98. Aceptadotras revisiónexternasinmodificaciones: 02.02.99.

Servicio de Neurología. ${ }^{a}$ Servicio de Hematología. Hospital Universitario Virgen del Rocío. Sevilla, España.

Correspondencia: Dr. Emilio Franco Macías. Servicio de Neurología. Hospital Universitario Virgen del Rocío. Avda de Manuel Siurot, s/n. E-41005 Sevilla.

(C) 1999, REVISTA DE NEUROLOGÍA mente tratamiento hipotensor con clortalidona y espironolactona. El parto fue inducido con oxitocina, sin incidencias, con nacimiento de un niño sano de bajo peso.

Dos semanas después del parto comenzó con cefaleas intensas sin respuesta a los analgésicos habituales. En las 48 horas previas a su ingreso presentaba fiebre de $38^{\circ} \mathrm{C}$, alteración del comportamiento y cuadro confusional. En la exploración inicial tenía tendencia al sueño, estaba desorientada en tiempo y espacio, presentaba febrícula y discreta rigidez de nuca. El fondo de ojo y el resto de pares craneales eran normales. No había asimetrías en la movilidad de miembros, los reflejos osteotendinosos eran simétricos, con respuestas plantares derecha flexora e izquierda indiferente. Cerebelo normal. El examen general, la auscultación cardíaca y de carótidas fueron normales, así como las cifras tensionales.

La analítica general, estudio de lípidos, ANA, ECG, Rx de tórax, ecocardiograma y estudio Doppler extracraneal fueron normales. En el estudio biológico de coagulación se detectó un anticoagulante lúpico. El LCR mostró 8 células ( $50 \%$ linfocitos), proteinorraquia de $230 \mathrm{mg} / \mathrm{dl}$, glucorraquia normal y estudio microbiológico negativo. La TAC craneal mostraba una hipodensidad bitalámica mal definida. La serie con contraste ponía de manifiesto un signo ‘delta vacío' a nivel de la vena de Galeno (Fig. 1). La RM practicada el día de su ingreso mostraba una lesión bitalámica hipointensa en $\mathrm{T}_{1}$ e hiperintensa en $\mathrm{T}_{2} \mathrm{y}$ en densidad protónica (Fig. 2). Existía además otra imagen de aspecto isquémico a nivel cerebeloso izquierdo. Se realizó un examen angiográfico (Fig. 3) de ambas carótidas y de la arteria vertebral izquierda que mostró un llenado muy tenue de la vena cerebral interna, vena de Galeno y seno recto en el sistema carotídeo derecho y ausencia de llenado de estas estructuras en el sistema carotídeo izquierdo. El resto del sistema venoso era permeable. Una RM de control realizada ocho días más tarde (Fig. 4) mostró en corte sagital una imagen hiperintensa en $\mathrm{T}_{1}$ que correspondía a la vena cerebral interna y vena de Galeno, sugestiva de trombosis. Se apreciaba, además, una lesión hiperintensa en $\mathrm{T}_{2}$ a nivel de vermis superior.

Inicialmente fue tratada con cefotaxima, ampicilina y corticosteroides, sustituyéndose por hipocoagulación con dicumarínicos en los días siguientes. En 72 horas mejoró llamativamente el nivel de conciencia y progresivamente en los siguientes días se mostró orientada, sin alteraciones cognitivas y sin focalidad neurológica. Fue dada de alta totalmente recuperada y en tratamiento con dicumarínicos. Tres meses después continúa asintomática.

\section{DISCUSIÓN}

Laexistenciade unaalteración delacoagulabilidadenelcontexto de un estado protrombótico (embarazo, puerperio, inmovilización) es el mecanismo patogénico propuesto en las trombosis venosas cerebrales [10].

Hasta en un $27 \%$ de pacientes con TVC se encuentran estados de hipercoagulabilidad [11] y ello obliga a realizar un estu- 


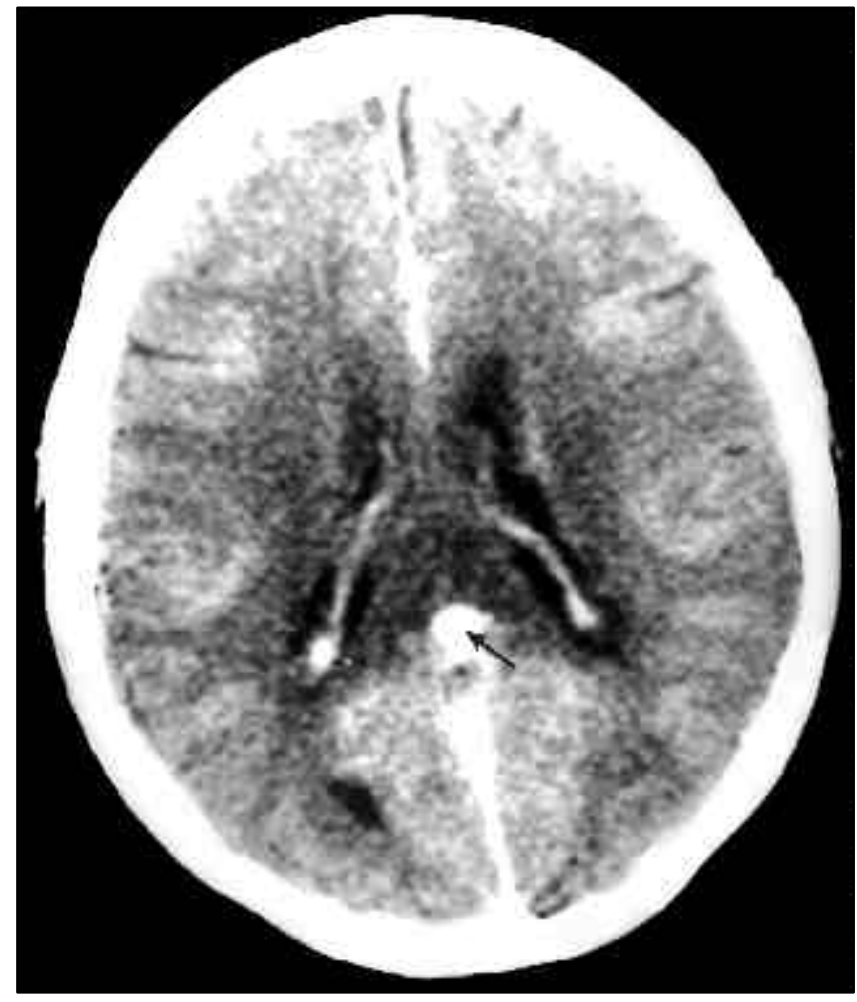

Figura 1. TAC con contraste. Se observa signo ‘delta vacío' a nivel de la vena de Galeno (flecha).

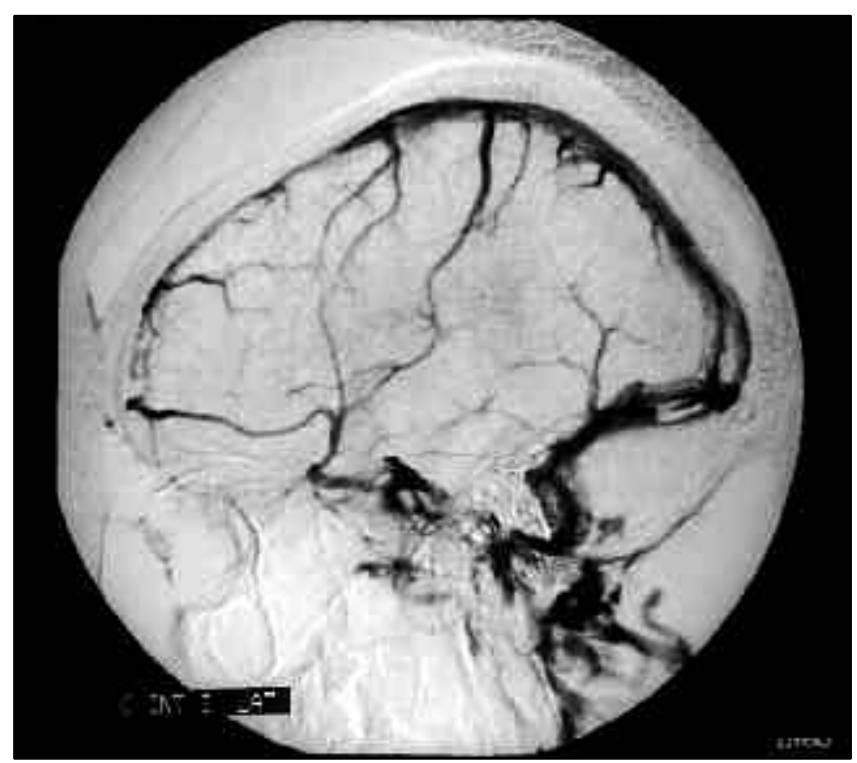

Figura 3. Angiografíacarotídea izquierda. En fases venosas se apreciauna ausencia de llenado de la vena cerebral interna, vena de Galeno y seno recto.

dio rutinario para anticuerpos antifosfolípidos, proteína S, proteína C, antitrombina III y factor V de Leiden $[10,12]$. El hallazgo más frecuente es la existencia de anticuerpos antifosfolipídicos, presentes en el 10\% de casos de TVC de las grandes series [11]. El porcentaje de trombosis venosa cerebral aséptica relacionada con causas obstétricas es muy variable en función del desarrollo económico de la población de procedencia de la serie. Así, en países occidentales se estima que la TVC

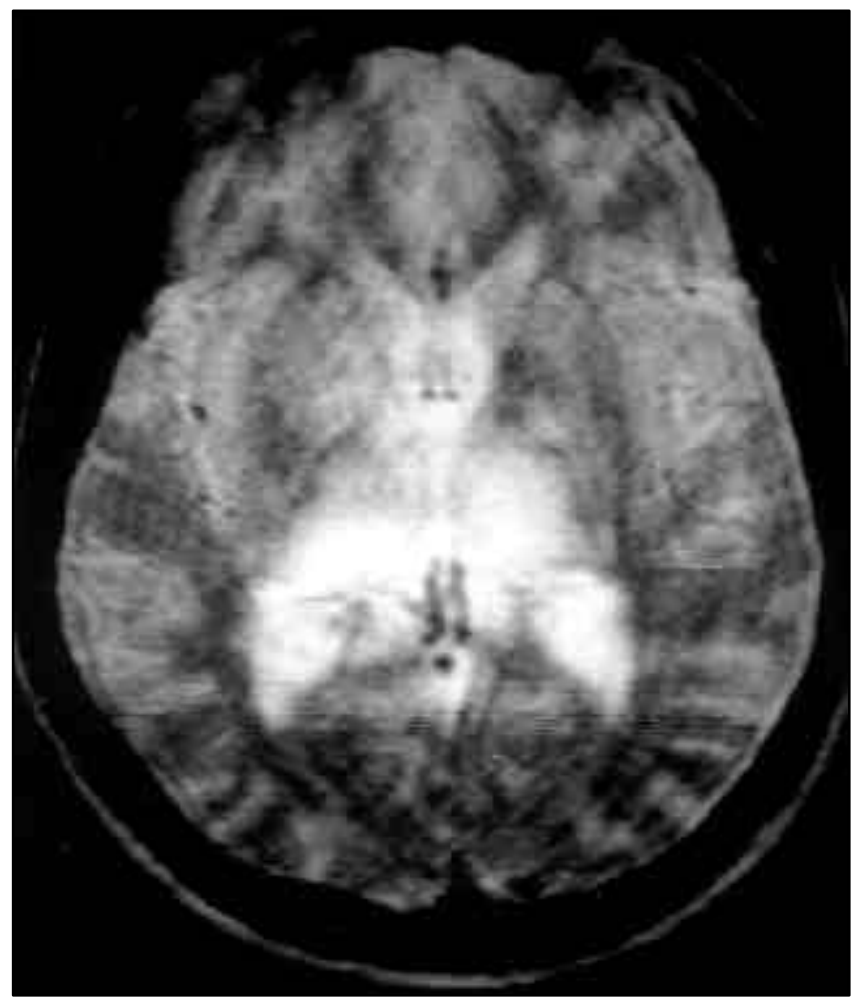

Figura 2. RM cerebral. En lasecuencia potenciada en densidad protónica se aprecia una hiperintensidad difusa y extensa que afecta a tálamo bilateralmente.

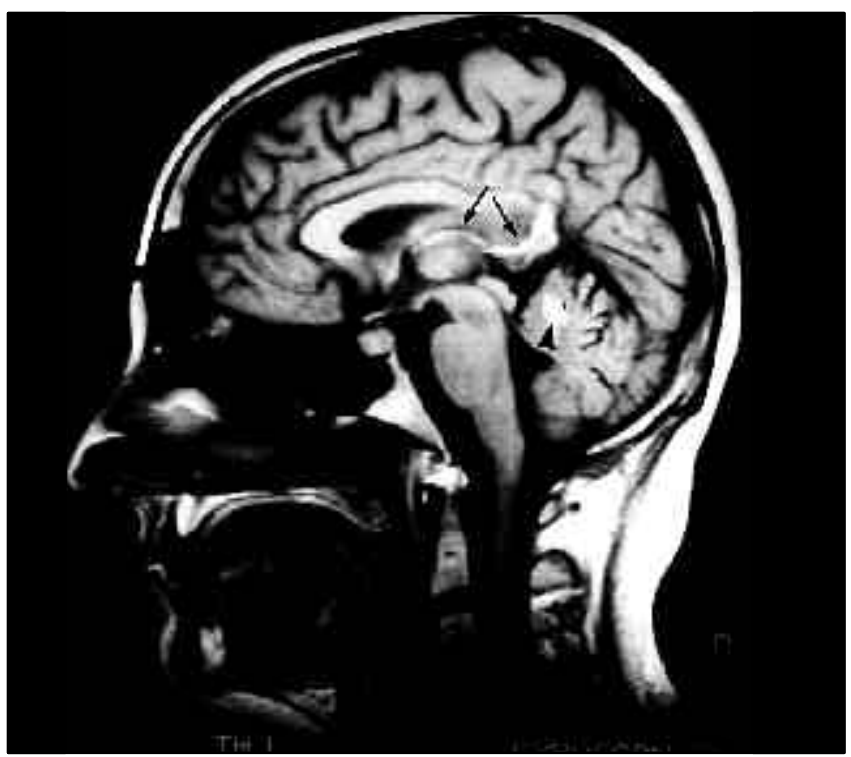

Figura 4. RM, corte sagital. En la secuencia potenciada en $T_{1}$ se observa una señal hiperintensa a nivel de la vena cerebral interna y vena de Galeno (flechas). Lesión a nivel del vermis cerebeloso (cabeza de flecha).

obstétrica supone del 1 al 10\% del total de TVC aséptica, siendo un factor etiológico más importante en las mujeres jóvenes de estos países la toma de anticonceptivos [1]. Por el contrario, en países más pobres, hasta el $60 \%$ de la TVC aséptica [13] se relaciona con causas obstétricas, y el puerperio constituye el período de mayor riesgo, especialmente en las tres primeras semanas tras el parto.

La TVC obstétrica tendría una presentación más aguda, una 
tendencia a mejorar o a estabilizarse antes, y un pronóstico más favorable. El seno longitudinal superior es su localización más frecuente y sólo en un 7,4\% de los casos la localización es aislada en el sistema venoso profundo [13].

Lapaciente debutó con cefalea, cuadroconfusional y fiebre, junto a discretas anomalías licuorales, sospechándose inicialmente una encefalitis. Esta forma de debut, poco indicativa de cuadro vascular, ya ha sido descrita por otros autores [6,9], y el cuadro confusional y la fiebre se han atribuido a la isquemia diencefálica. La pleocitosis e hiperproteinorraquia están condicionadas por el edema venoso y/o la necrosis hemorrágica $[4,9,14]$.

En un contexto clínico apropiado, la hipodensidad bitalámica se considera un indicador de alta sospecha de una trombosis de la vena de Galeno [4]. El diagnóstico diferencial debe plantearse principalmente con un infarto arterial talámico bilateral paramediano $[6,15]$. En la paciente que presentamos, una hiperdensidad a nivel de la vena de Galeno, con signo ‘delta vacío’ a ese nivel tras la administración de contraste, sugirieron el diagnóstico de TVCP.La aportación adicional de la RM en este caso fue la ulterior visualización de la trombosis a nivel de la vena de Galeno y vena cerebral interna. La ausencia de llenado del sistemavenosoprofundoenlaangiografíafuediagnósticade TVCP.

Es destacable el curso clínico benigno del proceso en esta paciente. Clásicamente, la TVCP se describe como un cuadro neurológico agudo, grave y de mal pronóstico $[1,4,9,16]$. En muchos casos, un importante descenso del nivel de conciencia con respuestas de descerebración y signos extrapiramidales preceden al fallecimiento del paciente, que tiene lugaren pocos días a pesar de llevar a cabo enérgicas medidas antiedema y del tratamiento anticoagulante. Si el paciente sobrevive, a menudo se describen secuelas importantes como alteración de funciones superiores, con desorientación, déficit de memoria reciente, abulia, mutismo acinético, parálisis de la mirada vertical y alteraciones extrapiramidales. En cambio, la paciente descrita tuvo un curso consistente en un cuadro confusional transitorio, de días de duración. Esta evolución benigna coincide con la de un reducido número de casos descritos previamente [1,5-7] y es atribuida al funcionamiento eficaz de una circulación venosa colateral compensadora[11].

\section{BIBLIOGRAFÍA}

1. Ameri A, Bousser MG. Cerebral venous thrombosis. Neurol Clin 1992; 10:87-111.

2. Garcin R, Pestel M. Sur un cas de thrombophlébite cérébrale post puerpérale (Contribution à l'étude de la pathologie veineuse de l'encéphale). Bull Soc Méd Hop Paris 1947; 63: 1012-21.

3. Brown JIM, Coyne TJ, Hurlbert RJ, Fehlings MG, Ter Brugge KG. Deep cerebral venous system thrombosis: case report. Neurosurgery 1993; 33: 911-3.

4. Madan A, Sluzewski M, van Rooij WJJ, Tijssen CC, Teepen JLJM. Thrombosis of the deep cerebral veins: CT and MRI findings with pathologic correlation. Neuroradiology 1997; 39: 777-80.

5. Bousser MG, Chiras J, Sauron B. Cerebral venous thrombosis. A review of 38 cases. Stroke 1985; 16: 199-213.

6. Silburn PA, Sandstrom PA, Staples C, Mowat P, Boyle RS. Deep cerebral venous thrombosis presenting as an encephalitic illness. Postgrad Med J 1996; 72: 355-7.

7. Hanigan WC, Rossi LJ, Mc Lean JM, Wright RM. MRI of cerebral vein thrombosis in infancy. A case report. Neurology 1986; 36: 1354-6.

8. Oguz M, Aksungur EH, Soyupak SK, Yildirim AU. Vein of Galen and sinus thrombosis with bilateral thalamic infarcts in sickle cell anaemia:

\section{TROMBOSIS VENOSA}

\section{CEREBRAL PROFUNDA PUERPERAL}

Resumen. Caso clínico. Dos semanas después de un parto inducido con oxitocina, una mujer primípara de 29 años comenzó con cefalea, fiebre y confusión. La TAC de cráneo mostró una hipodensidad bitalámica difusa y un signo 'delta vacío' a nivel de la vena de Galeno. El estudio angiográfico y una RM de cráneo demostraron la existencia de una trombosis venosa cerebral profunda (TVCP). Se detectó un anticoagulante lúpico y la enferma fue tratada con dicumarínicos, siguiendo una evolución favorable con recuperación completa en pocos días. Conclusiones. La TVCP aislada es muy infrecuente. La isquemia bitalámica extensa en el contexto de un estado protrombótico bien conocido, como el embarazo y el puerperio, debe hacer sospechar una trombosis de la vena de Galeno. La TVCP se considera una entidad grave, con alta mortalidad o graves secuelas. El curso clínico benigno de la TVCP es infrecuente y se ha relacionado con el desarrollo de circulación venosa colateral eficaz [REV NEUROL 1999; 29: 722-4].

Palabras clave. Anticoagulante lúpico. Infarto talámico. Puerperio. Trombosis venosa cerebral profunda.
CT follow-up and angiographic demonstration. Neuroradiology 1994; 36: $155-6$.

9. Milandre L, Pellissier JF, Vincentelli F, Khalil R. Deep cerebral venous system thrombosis in adults. Eur Neurol 1990; 30: 93-7.

10. Zuber M, Toulon P, Marnet L, Mas JL. Factor V Leiden mutation in cerebral venous thrombosis. Stroke 1996; 27: 1721-3.

11. Daif A, Awada A, Al-Rajeh S, Abduljabbar M, Rahman A, Obeid T, et al. Cerebral venous thrombosis in adults. Stroke 1995; 26: 1193-5.

12. Mokri B, Jack CR, Petty GW. Pseudotumor syndrome associated with cerebral venous sinus occlusion and antiphospholipid antibodies. Stroke 1993; 24: 469-72.

13. Cantú $C$, Barinagarrementeria $F$. Cerebral venous thrombosis associated with pregnancy and puerperium. Stroke 1993; 24: 1880-4.

14. Nishimura R, Stepanek D, Howieson J, Hammerstad J. Internal cerebral vein thrombosis. Arch Neurol 1982; 39: 439-40.

15. Bell A, Davids WL, Osborn AG, Harnsberger HR. Bithalamic hyperintensity on $\mathrm{T}_{2}$-weighted MR: vascular causes and evaluation with MR angiography. Am J Neuroradiol 1994; 15: 893-9.

16. Haley EC, Brashear HR, Barth JT, Cail WS, Kassell NF. Deep cerebral venous thrombosis. Arch Neurol 1989; 46: 337-40.

\section{TROMBOSE VENOSA}

\section{CEREBRAL PROFUNDA PUERPERAL}

Resumo. Caso clínico. Duas semanas depois de um parto induzido com oxitocina, uma mulher primípara de 29 anos começou com cefaleias, febre e confusão. A TAC de crânio mostrou uma hipodensidade bitalâmica difusa e um sinal 'delta vazio' ao nível da veia de Galeno. O estudo angiográfico e uma RM de crânio demostraram a existência de uma trombose venosa cerebral profunda (TVCP). Detectou-se um anticoagulante lúpico e a doente foi tratada com dicumarínicos, seguindo uma evolução favorável com recuperação completa em poucos dias. Conclusões. A TVCP isolada é muito infrequente. A isquémia bitalâmica extensa no contexto de estado protrombótico bem conhecido, como a gravidez ou o puerpério deve fazer suspeitar de uma trombose da veia de Galeno. A TVCP é considerada uma entidade grave, com elevada mortalidade ou graves sequelas. O curso clínico benigno da TVCP é infrequente e relacionou-se com o desenvolvimento de circulação venosa colateral eficaz [REV NEUROL 1999; 29: 722-4].

Palavras chave. Anticoagulante lúpico. Enfarte talâmico. Puerpério. Trombose venosa cerebral profunda. 